\title{
APROVEITAMENTO DE RESÍDUOS DE MADEIRA DE PINUS E BAGAÇO DE CANA DE AÇÚCAR PARA PRODUÇÃO DE CHAPAS DE MADEIRA AGLOMERADO
}

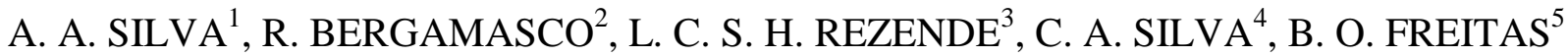 \\ ${ }^{1}$ Universidade Estadual de Londrina, Departamento de Engenharia de Edificações e Saneamento \\ ${ }^{2}$ Universidade Estadual de Maringá, Departamento de Engenharia Química \\ ${ }^{3}$ Universidade Estadual de Maringá, Departamento de Engenharia Mecânica \\ ${ }^{4}$ Universidade Estadual de Maringá, Departamento de Engenharia Química \\ ${ }^{5}$ Universidade Tecnológica Federal do Paraná, Departamento de Engenharia Ambiental \\ E-mail para contato: andressa_algayer@hotmail.com
}

RESUMO - O meio ambiente vem sendo utilizado como fonte de matéria prima para o setor agroindustrial. Os resíduos sólidos, como as sobras das indústrias madeireiras e bagaço de cana de açúcar possui baixo aproveitamento como material reciclável. Neste contexto, uma das maneiras de reaproveitamento destes resíduos é a confecção de chapas de madeira aglomerada. O objetivo deste trabalho foi estudar as diferentes proporções de incorporação das fibras do bagaço de cana e maravalhas de pinus na produção de chapas aglomeradas de madeira. $\mathrm{O}$ experimento foi composto por cinco tratamentos, variando a incorporação em T1 $100 \%$ e $0 \%$, T2 $=75 \%$ e $25 \%$, T3 $=50 \%$ e $50 \%, \mathrm{~T} 4=25 \%$ e $75 \%$ e $\mathrm{T} 5=0 \%$ e $100 \%$ de maravalha e bagaço de cana respectivamente. A quantidade de resina foi de $12 \%$ e a densidade inicial do painel de $0,7 \mathrm{~g} / \mathrm{cm}^{3}$. Foi realizada uma caracterização prévia das partículas. A avaliação do painel foi de acordo com a ABNT NBR 14810-2 (2006). Pode-se considerar que o melhor resultado obtido, quanto às análises físicas foi para T4. A análise mecânica foi realizada apenas para o T4, contudo, o valor do modulo de ruptura não se enquadrou na norma, porém valores semelhantes foram encontrados na literatura.

\section{INTRODUÇÃO}

O meio ambiente vem sendo utilizado como fonte de matéria prima para desenvolvimento do setor agroindustrial, e os seus resíduos e produtos que não são usados no processo produtivo são descartados em locais de "área de despejo" (FIORELLI et al, 2011).

Atualmente tem-se dado enfoque maior às questões ambientais, à gestão adequada dos resíduos bem como ao reúso dos mesmos. Segundo Mota (2006) o reaproveitamento dos resíduos surge como uma alternativa, pois esta prática traz benefícios ambientais, diminuindo a utilização de recursos naturais além de reduzir a poluição causada pela destinação final inadequada.

Segundo (BRITO et al, 2005) as sobras provenientes das indústrias madeireiras no Brasil são resíduos que são pouco utilizados e são gerados em grandes quantidades. Iwakiri (2005) aponta que o processamento para a redução do volume das árvores retiradas das florestas, geram muitos tipos de 


\section{9 a 22 de outubro de 2014 \\ Florianópolis/SC}

partículas de madeira, com formas e tamanhos diferentes, possibilitando a geração de novos produtos, através do reaproveitamento, como por exemplo, os painéis aglomerados de madeira.

Dentre os tipos de resíduos madeireiros, dois são mais significativos, sendo as maravalhas e as cascas de madeira. As maravalhas podem ser utilizadas isoladamente ou combinado com outros materiais para a produção de chapas aglomerados de madeira, pois apresentam boas características para esta finalidade (BRITO, 1995).

Outro resíduo que possui grande geração no Brasil e que vem sendo pouco explorado é o bagaço da cana de açúcar (BASTTINELLI et al. 2008), sendo a queima para a geração de energia a principal destinação.

Devido à grande produção e principalmente às propriedades química e física do bagaço de cana de açúcar, alguns estudos mostram destinações como: produção de ração animal, na indústria química, na fabricação de papel e papelão, na própria produção de etanol utilizando o bagaço e a palha, na produção de biomassa microbiana, na construção civil como material agregado, e em aglomerado de madeiras (SILVA, 2010).

Carvalho (2012) destaca que o principal motivo de utilizar o bagaço da cana de açúcar para a produção de chapas de aglomerado é a possibilidade de substituição de madeiras provenientes de florestas plantadas.

Este trabalho tem como objetivo estudar diferentes proporções de incorporação do bagaço da cana de açúcar e partículas de madeira com aplicação de $12 \%$ de resina, para produção de chapa de madeira aglomerado.

\section{METODOLOGIA}

A coleta do resíduo de madeira (maravalha) foi realizada na empresa Cia do Pinus localizada na cidade de Londrina-PR e o bagaço da cana de açúcar foi coletado na Usina Santa Terezinha na cidade de Maringá-PR. Ambos resíduos foram doados pelas respectivas empresas, sendo coletado manualmente $50 \mathrm{Kg}$ de cada resíduo. A maravalha foi triturada para diminuir o tamanho das partículas. $\mathrm{O}$ bagaço de cana de açúcar foi peneirado para retirada do excesso do pó a fim de diminuir as interferências no processo de produção das chapas.

Para a caracterização dos resíduos, foram realizadas análises físicas como teor de umidade, utilizando a metodologia do Departamento Nacional de Estradas de Rodagem - DNER 213/1994 e granulometria de acordo com a norma NBR 7181/1984. Em relação à análise de superfície foi realizado o teste de microscopia eletrônica de varredura (MEV) com o Equipamento Superscan SS 550 marca Shimadzu.

O experimento foi composto por cinco tratamentos em triplicata, utilizando um delineamento inteiramente casualisado totalizando 15 painéis, onde foram analisadas diferentes porcentagens de incorporação do bagaço da cana de açúcar (Tabela 1). A porcentagem da resina de ureia formaldeído 
foi fixada em $12 \%$ bem como a densidade do painel aglomerado em $0,7 \mathrm{~g} / \mathrm{cm} 3$. Esses dados foram baseados em estudos anteriores (MENDES, 2012 e IWAKIRI, 2005).

Tabela 1 - Tratamentos em função das diferentes porcentagens de bagaço da cana de açúcar e partículas de madeira

\begin{tabular}{ccccc}
\hline Tratamentos & $\begin{array}{c}\text { Densidade do painel } \\
(\mathrm{g} / \mathrm{cm} 3)\end{array}$ & $\begin{array}{c}\text { Bagaço de Cana de Açúcar } \\
(\%)\end{array}$ & Madeira $(\%)$ & Teor de resina $(\%)$ \\
\hline \hline T1 & 0,7 & 0 & 100 & 12 \\
T2 & 0,7 & 25 & 75 & 12 \\
T3 & 0,7 & 50 & 50 & 12 \\
T4 & 0,7 & 75 & 25 & 12 \\
T5 & 0,7 & 100 & 0 & 12 \\
\hline
\end{tabular}

Os cálculos das quantidades de partículas de madeira e bagaço de cana de açúcar bem como de resina e seus aditivos foram efetuados com base no peso seco das partículas (0\% de umidade), apresentados na Tabela 2 .

Tabela 2 - Composição dos painéis com resina de ureia formaldeído

\begin{tabular}{|c|c|c|c|c|c|c|c|c|c|c|c|c|}
\hline \multirow[b]{2}{*}{ Tratam. } & \multirow[b]{2}{*}{$\begin{array}{l}\text { Volume } \\
\left(\mathrm{cm}^{3}\right)\end{array}$} & \multicolumn{3}{|c|}{ PAINEL } & \multicolumn{2}{|c|}{ PARTÍCULAS } & \multicolumn{2}{|c|}{ RESINA (UF) } & \multicolumn{2}{|c|}{$\begin{array}{c}\text { IMPERMEABILIZ } \\
\text { ANTE } \\
\end{array}$} & \multicolumn{2}{|c|}{ CATALISADOR } \\
\hline & & $\begin{array}{c}\text { Massa } \\
\text { painel } \\
(\mathrm{g})\end{array}$ & $\begin{array}{c}\text { Massa das } \\
\text { partículas } \\
\text { secas no } \\
\text { painel }(\mathrm{g})\end{array}$ & $\begin{array}{c}\text { Massa com } \\
12 \% \text { de } \\
\text { umidade } \\
(\mathrm{g})\end{array}$ & $\begin{array}{c}\text { Bagaço } \\
\text { de } \\
\text { Cana } \\
\text { Peso (g) }\end{array}$ & $\begin{array}{l}\text { Madeira } \\
\text { Peso (g) }\end{array}$ & $\begin{array}{c}\text { Sólidos } \\
\text { (g) na } \\
\text { Resina }\end{array}$ & $\begin{array}{c}\text { Resina } \\
\text { na } \\
\text { forma } \\
\text { líquida } \\
\text { (g) }\end{array}$ & $\begin{array}{c}\text { Sólidos } \\
\text { (g) na } \\
\text { Parafina }\end{array}$ & $\begin{array}{c}\text { Parafina } \\
\text { na } \\
\text { forma } \\
\text { líquida } \\
\text { (g) }\end{array}$ & $\begin{array}{c}\text { Sólidos (g) } \\
\text { de } \\
\left(\mathrm{NH}_{4}\right)_{2} \mathrm{SO}_{4}\end{array}$ & $\begin{array}{c}\text { Solução } \\
\text { Catalisador } \\
\text { (g) }(25 \%)\end{array}$ \\
\hline T1 & 4538 & 3176 & 2541 & 2845,92 & 0 & 2845,92 & 304,92 & 462 & 25,41 & 49,82 & 9,15 & 36,59 \\
\hline $\mathrm{T} 2$ & 4538 & 3176 & 2541 & 2845,92 & 711,48 & 2134,44 & 304,92 & 462 & 25,41 & 49,82 & 9,15 & 36,59 \\
\hline T3 & 4538 & 3176 & 2541 & 2845,92 & 1423 & 1422,96 & 304,92 & 462 & 25,41 & 49,82 & 9,15 & 36,59 \\
\hline $\mathrm{T} 4$ & 4538 & 3176 & 2541 & 2845,92 & 2134,4 & 711,48 & 304,92 & 462 & 25,41 & 49,82 & 9,15 & 36,59 \\
\hline T5 & 4538 & 3176 & 2541 & 2845,92 & 2845,9 & 0 & 304,92 & 462 & 25,41 & 49,82 & 9,15 & 36,59 \\
\hline
\end{tabular}

Os resíduos de maravalha e bagaço de cana foram pesados e colocados em um recipiente, respeitando as proporções e pesos de acordo com os tratamentos propostos anteriormente, Tabela 2. A quantidade de resina foi pesada bem como a quantidade de catalisador sendo que este foi diluído em água e misturado à resina. A resina já com o catalisador junto com a emulsão de parafina foi adicionada à maravalha e ao bagaço de cana previamente distribuído em um recipiente e então misturado manualmente. Esta mistura foi distribuída sobre os moldes de metal quadrados de dimensões 55 x 55 x 1,5 (cm) formando um colchão de partículas.

Então foi realizada uma pré - prensagem, a fim de reduzir o volume de colchão de partículas e a perda do mesmo durante o transporte para a prensa. O molde foi levado para prensagem à quente em um equipamento da marca SIRMAS pertencente à empresa Madeireira Ibiporã localizada no município de Ibiporã-PR.

Os painéis foram prensados a uma temperatura de $160{ }^{\circ} \mathrm{C}$ (WEBER, 2011) e pressão de aproximadamente de $50 \mathrm{kgf} / \mathrm{cm} 2$ por um tempo de 10 minutos, após a prensagem os painéis foram deixados à temperatura ambiente até alcançar a temperatura de equilíbrio.

Os painéis foram cortados e analisados conforme estabelecido pela norma NBR 14810-3 (2006). Pode-se verificar na Tabela 3, as análises físicas e mecânicas, o tamanho de cada corpo de 
prova, quantidade de amostra por painel e quantidade de amostra por tratamento, segundo os parâmetros exigidos pela norma.

Tabela 3 - Dimensões e quantidade de amostras dos corpos de prova para ensaio de avaliação dos painéis de madeira aglomerada

\begin{tabular}{ccccc}
\hline Avaliações físico-mecânicas & Comprimento $(\mathrm{mm})$ & Largura $(\mathrm{mm})$ & $\begin{array}{c}\text { Quantidade de amostras } \\
\text { por painel }\end{array}$ & $\begin{array}{c}\text { Quantidade de amostras } \\
\text { por tratamento }\end{array}$ \\
\hline \hline Densidade & 50 & 50 & 6 & 18 \\
Teor de Umidade & 50 & 50 & 6 & 18 \\
Absorção de água & 25 & 25 & 6 & 18 \\
Inchamento em espessura & 25 & 25 & 6 & 18 \\
Flexão estática & 250 & 50 & 6 & 18 \\
\hline
\end{tabular}

Fonte: Adaptado da Norma Brasileira ABNT NBR 14810 - 3 (2006).

Para a análise mecânica de flexão estática foi utilizado a maquina universal de ensaios da marca SHIJIN, modelo WDW-100E pertencente à Universidade Tecnológica Federal do Paraná, câmpus Londrina, no laboratório de Engenharia de Materiais.

Para comparação dos resultados, foi utilizado o programa estatístico STATSOFT STATISTICA versão 8.0. Foi realizada a Análise de Variância (ANOVA) e o teste de comparação de médias, teste Tukey, considerando $95 \%$ de confiança, sendo significativo um p-valor < 0,05. A análise estatística foi efetuada para verificar as diferenças significativas das incorporações dos resíduos nos parâmetros avaliados.

\section{RESULTADOS}

\subsection{Análise das Partículas de Bagaço de Cana de Açúcar e Madeira de Pinus sp}

A análise granulométrica para as partículas de bagaço de cana de açúcar mostrou que a maior parte das partículas encontraram-se na peneira de $9,5 \mathrm{~mm}$ e $4,75 \mathrm{~mm}$, correspondendo à $26,4 \%$ e $26,1 \%$ respectivamente. Para as partículas da maravalha de pinus houve uma concentração na peneira de diâmetro $1,18 \mathrm{~mm}$ correspondendo à aproximadamente $80 \%$ do total. A curva granulométrica de cada resíduo encontra-se na Figura 1.
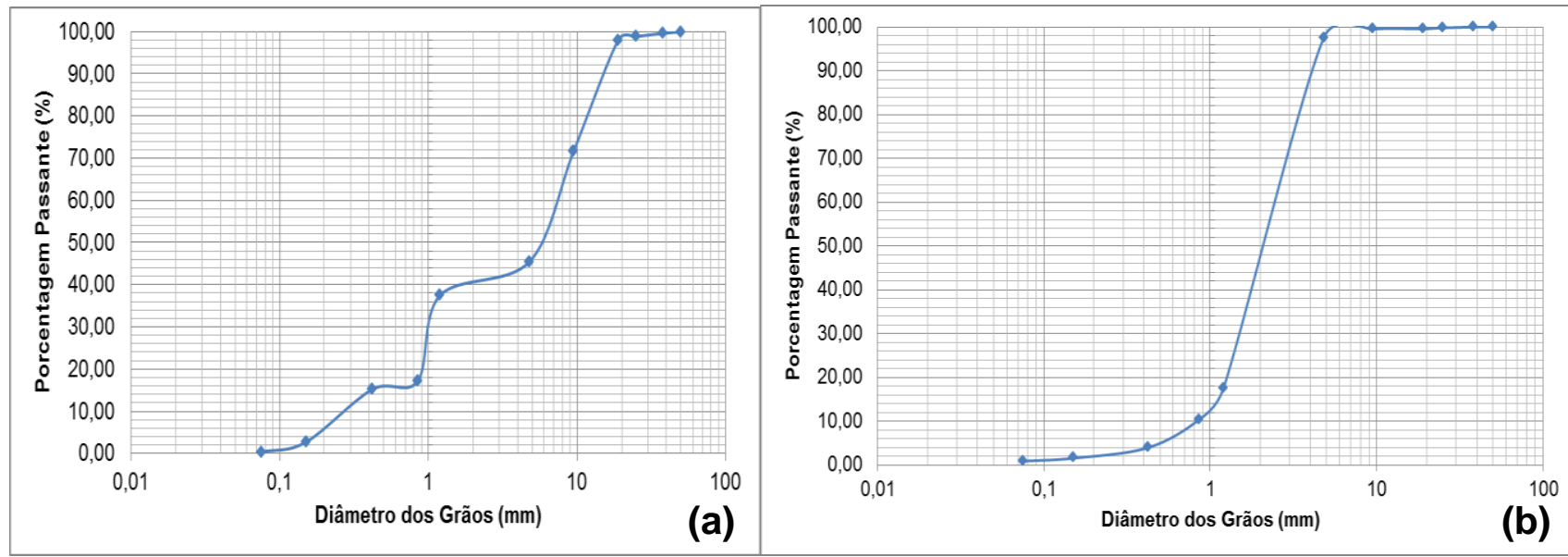


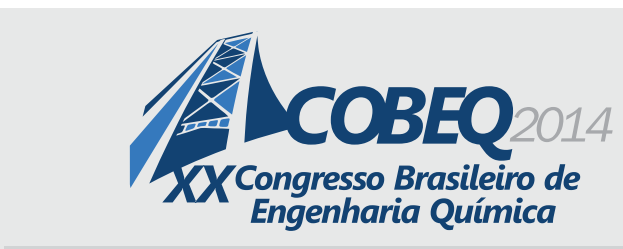

19 a 22 de outubro de 2014

Florianópolis/SC

Figura 1 - Curva granulométrica. a) bagaço de cana de açúcar e b) maravalha de pinus

De acordo com a análise de umidade realizada verificou que os resíduos de maravalha e bagaço de cana apresentaram uma média de $10,86 \%$ e $10,48 \%$, respectivamente.

Através da análise de microscopia eletrônica por varredura (MEV) foi possível visualizar as estruturas das partículas de bagaço de cana de açúcar e madeira de pinus, Figuras 2 e 3 respectivamente.

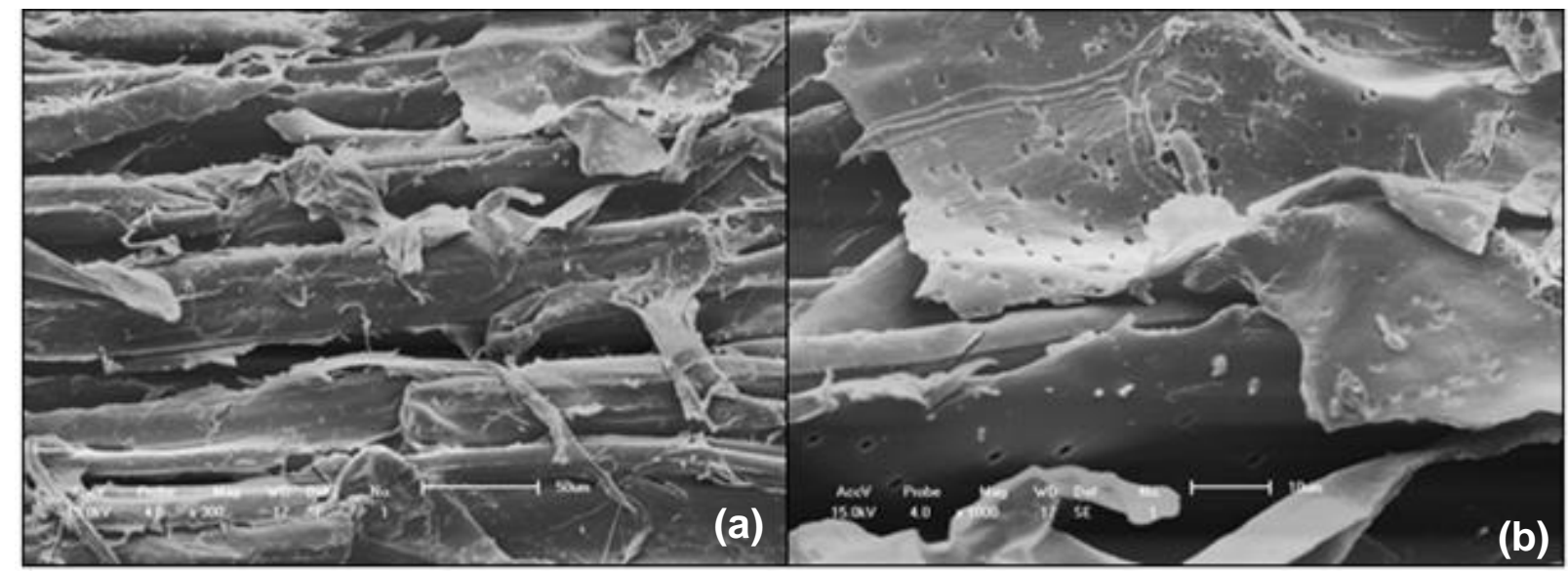

Figura 2 - Fotomicrografias por MEV do resíduo de bagaço de cana de açúcar. a) 300x e b) 1000x

Com as imagens do bagaço de cana de açúcar foi possível visualizar a estrutura das fibras microscopicamente. Foi observado que as fibras possuem direcionamento longitudinal e listras paralelas, além disto, apresentou uma superfície irregular e porosa, podendo ser observada nas amplitudes de 300 x e 1000 x, ilustradas na Figura 2.

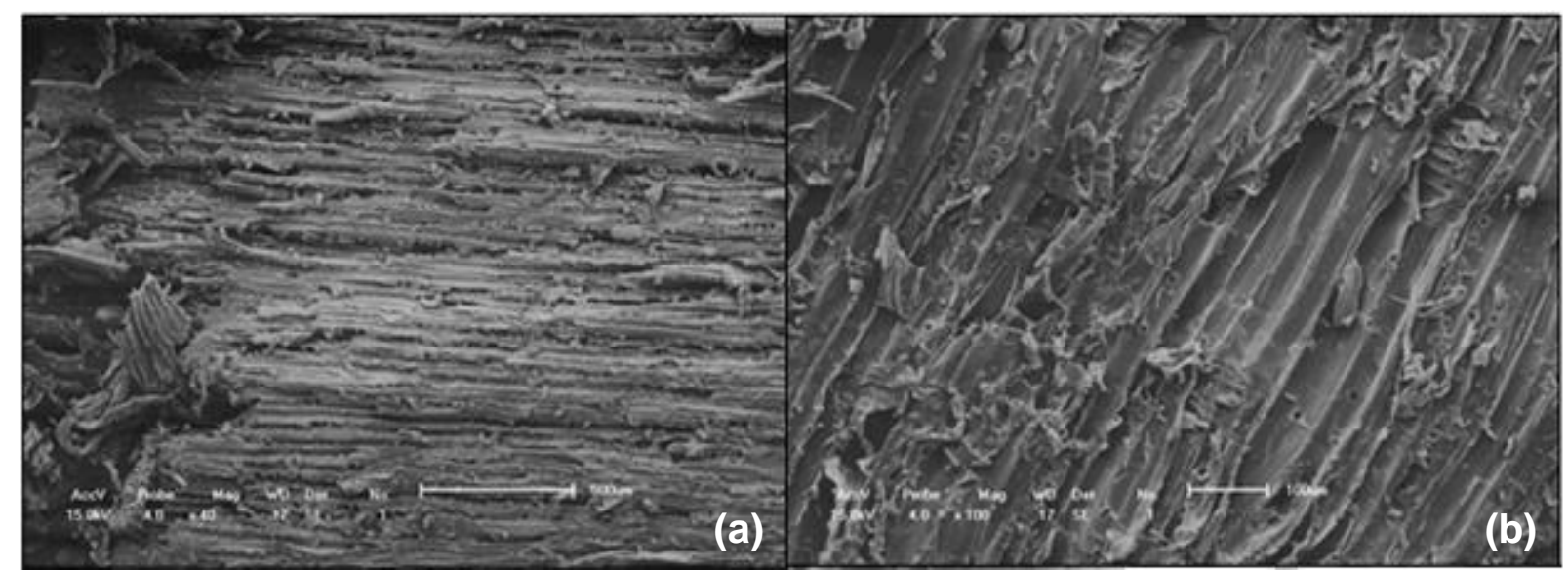

Figura 3 - Fotomicrografias por MEV do resíduo de maravalha de pinus. a) 40 x b) $100 \mathrm{x}$

Foi possível notar, através da Figura 3 que a maravalha possui um perfil completamente irregular. Na ampliação de 40 x (a) em especifico, pode-se perceber um rompimento das fibras 
ocasionado provavelmente pelo processamento da madeira. E na ampliação de 100 x nota-se a presença de poros na superfície.

\subsection{Análise das Chapas de Madeira Aglomerada}

As médias dos resultados das análises físicas do painel de madeira aglomerada referente à densidade, teor de umidade, inchamento em espessura (IE) após 2 horas e 24 horas e absorção de água (AA) após 2 horas e 24 horas, estão expressos na Tabela 4.

Tabela 4 - Média das análises físicas do painel.

\begin{tabular}{|c|c|c|c|c|c|c|}
\hline Tratamento & $\begin{array}{c}\text { Média da } \\
\text { Densidade } \\
(\mathrm{kg} / \mathrm{m} 3)\end{array}$ & $\begin{array}{c}\text { Teor de } \\
\text { umidade (\%) }\end{array}$ & $\begin{array}{c}\text { Inchamento em } \\
\text { espessura (\%) após } 2 \\
\text { horas } \\
\end{array}$ & $\begin{array}{c}\text { Inchamento em } \\
\text { espessura (\%) após } \\
24 \text { horas }\end{array}$ & $\begin{array}{l}\text { Absorção de água } \\
(\%) \text { após } 2 \text { horas }\end{array}$ & $\begin{array}{l}\text { Absorção de água } \\
(\%) \text { após } 2 \text { horas }\end{array}$ \\
\hline 1 & $338,47^{\mathrm{c}}$ & $14,70^{\mathrm{a}}$ & $7,43^{\mathrm{b}}$ & $9,74^{\mathrm{a}}$ & $72,13^{\mathrm{c}}$ & $86,50^{\mathrm{c}}$ \\
\hline 2 & $366,64^{\mathrm{c}}$ & $15,08^{\mathrm{a}}$ & $10,21^{\mathrm{c}}$ & $12,13^{\mathrm{a}}$ & $84,74^{\mathrm{c}}$ & $104,76^{\mathrm{d}}$ \\
\hline 3 & $355,33^{\mathrm{c}}$ & $14,67^{\mathrm{a}}$ & $8,78^{\mathrm{bc}}$ & $10,93^{\mathrm{a}}$ & $77,84^{\mathrm{c}}$ & $110,96^{\mathrm{d}}$ \\
\hline 4 & $447,42^{\mathrm{a}}$ & $15,46^{\mathrm{a}}$ & $6,61^{\mathrm{ab}}$ & $11,26^{\mathrm{a}}$ & $35,75^{\mathrm{b}}$ & $67,67^{\mathrm{b}}$ \\
\hline 5 & $412,52^{\mathrm{b}}$ & $15,20^{\mathrm{a}}$ & $3,93^{\mathrm{a}}$ & $11,83^{\mathrm{a}}$ & $20,34^{\mathrm{a}}$ & $47,59^{\mathrm{a}}$ \\
\hline
\end{tabular}

(1) Dentro de uma mesma coluna, as médias seguidas por mesma letra não diferem estatisticamente entre si, pelo teste de Tukey a 5\% de nível de significância.

Com base nos dados da Tabela 4, verificou-se que os valores de densidade ficaram abaixo do valor pré-estabelecido de $0,7 \mathrm{~g} / \mathrm{cm}^{3}$, ou seja, $700 \mathrm{Kg} / \mathrm{m}^{3}$. O maior valor médio encontrado foi para o tratamento 4 o qual possui $75 \%$ de bagaço de cana de açúcar e $25 \%$ de partículas de maravalha com $447,42 \mathrm{Kg} / \mathrm{m} 3$, o que pode ser comprovado pela análise estatística aplicada. Contudo nenhum tratamento encontrou-se na faixa pré-estabelecida pela norma brasileira ABNT NBR 14810-2 (2006), qual classifica um painel de madeira aglomerada como média densidade na faixa que compreende os valores de $551 \mathrm{Kg} / \mathrm{m} 3$ até $750 \mathrm{Kg} / \mathrm{m} 3$.

Em relação ao teor de umidade, a Norma 14810-2 de (2006) estabelece que a umidade presente em chapas de madeira aglomerada não deve ser menor que 5\% e não deve ultrapassar $11 \%$. De acordo com a Tabela 4, pode-se observar que nenhum tratamento se enquadrou nos parâmetros estabelecidos pela norma. O maior valor de teor de umidade obtido foi para o tratamento 4 com $15,46 \%$ e o menor valor obtido foi para o tratamento 1 com $14,70 \%$, porém nenhum dos tratamentos diferiram estatisticamente entre si. $\mathrm{O}$ alto teor de umidade nos painéis pode ter ocorrido, devido a falta de um pré-tratamento adequado das partículas para diminuir o teor de umidade inicial e de uma câmara climatizada, com controle de umidade e temperatura para a estabilização do painel após prensagem.

As porcentagens de inchamento em espessura (IE) encontram-se na Tabela 4. O menor valor encontrado para IE após 2 horas foi o tratamento 5 com 3,93\% e o maior valor foi para o tratamento 2 com 10,32\%, porém o tratamento 4 apresentou diferença estatística do tratamento 5 . Após 24 horas observou que o maior valor de IE ocorreu para o tratamento 2 com 12,13\% e o menor valor foi para o 


\section{9 a 22 de outubro de 2014 \\ Florianópolis/SC}

tratamento 1 com 9,74\%, entretanto em relação a este parâmetro os tratamentos não diferiram estatisticamente entre si.

A ABNT NBR 14810-2 (2006) estabelece valores de inchamento em espessura máximo, após duas horas de imersão, em 8\%. Logo comparando os resultados obtidos com a norma pode-se verificar que os tratamentos 1,4 e 5 se enquadram dentro do limite estabelecido. Esta norma não prevê limites de inchamento em espessura após 24 horas. Ainda pode-se observar que os tratamentos 4 e 5 com $75 \%$ de incorporação de bagaço de cana e 100\% de bagaço de cana de açúcar respectivamente, tiveram maior densidade e o menor inchamento em espessura. Sendo então os melhores tratamentos encontrados.

Verificam-se na Tabela 4 os resultados obtidos para absorção de água (AA), após 2 horas e após 24 horas de imersão. O menor valor de AA para imersão após 2 horas foi o tratamento 5 seguido do tratamento 4, conforme mostrado pela análise estatística na Tabela 4, e o maior valor foi para o tratamento 2, não diferindo estatisticamente dos tratamentos 1 e 3. Após 24 horas, o menor valor encontrado foi para o tratamento 5 e o maior valor foi para o tratamento 3 .

A norma brasileira NBR 14810-2 não apresenta valores limites para a análise de AA, porém nota-se que para os tratamentos 4 e 5 que tiveram maior densidade, obtiveram, tanto para imersão após 2 horas quanto para 24 horas, os menores valores de absorção de água.

Portanto a análise estatística aplicada nas variáveis físicas apresentadas acima, permite inferir que o tratamento 4 foi o melhor tratamento encontrado, já que obteve o maior valor de densidade. No que se refere aos valores de teor de umidade e inchamento em espessura após 2 horas, que são os parâmetros físicos que a norma ABNT NBR 14.810-2 (2006) estabelece limites, o tratamento 5 foi o melhor tratamento encontrado para estes parâmetros, porém não difere estatisticamente do tratamento 4.

Quanto a análise mecânica de flexão estática foi escolhido o tratamento 4, por possuir o maior valor de densidade comparado aos outros tratamentos. A ABNT NBR 14810-2 (2006) estipulou o limite mínimos de $16 \mathrm{MPa}$ para o modulo de ruptura (MOR). O valor médio encontrado para o tratamento 4 foi de 1,87 MPa, não atendendo o estabelecido pela norma. Provavelmente isto deve ter ocorrido devido à baixa densidade encontrada para o tratamento 4 no valor de 447,42 Kg/m3, ou seja, quanto maior a densidade do painel aglomerado de madeira maior a força necessária para acontecer a ruptura. O valor médio de MOE para o tratamento 4 foi de 897,13 MPa, porém não há valores limites indicados pela norma 14810-2 (2006).

\section{CONCLUSÕES}

Pôde-se concluir que os parâmetros físicos e mecânicos não se enquadraram no estabelecido na norma ABNT NBR 14810 e, portanto ainda é necessário o ajuste de parâmetros de confecção dos painéis de madeira aglomerada com a incorporação do resíduo de bagaço de cana, como temperatura, pressão e tempo de prensagem. Além disto, um dos principais fatores que deve ser otimizado é o ajuste do teor de umidade inicial das partículas, bem como a distribuição da resina durante a mistura 
buscando deixar o painel o mais homogêneo possível, pois isso influencia na qualidade do painel formado.

Contudo, o reaproveitamento de resíduos sólidos vem se tornando cada vez mais viável e para isto é necessários estudos que avaliem a efetividade do sistema. Esta prática tem como consequência um ganho econômico, e principalmente ambiental, pois reduz os impactos gerados pela disposição inadequada destes resíduos, visando por tanto a sustentabilidade.

\section{REFERÊNCIAS}

ABNT - NBR 14.810-2. Chapas de madeira aglomerada. Parte 2: Requisitos. Rio de Janeiro, 2006.

ABNT - NBR 14.810-3. Chapas de madeira aglomerada. Parte 3: Métodos de ensaio. Rio de Janeiro, 2006.

BASTINELLI, R.A.G.; MARCILIO, C.; LAHR, F.A.R. Emprego do bagaço da cana de açúcar (Saccharum officinarum) e das folhas caulinares do bambu da espécie Dendrocalamus giganteus na produção de chapas de partículas. Pesq. e Tecno., v. 5, p. 297-305, 2008.

BRITO, E.O. Produção de chapas de partículas de madeira a partir de maravalhas de Pinus elliotti plantado no Sul do Brasil. Tese (Doutorado em Engenharia Florestal) - Setor de Ciências Agrárias, Universidade Federal do Paraná, Curitiba. 127f, 1995.

BRITO, E.O. et al. Chapas de madeira aglomerada de uma camada de Pinus elliottii Engelm com a adição das cascas de Eucalyptus pellita F. Muell. Rev. Cerne., v. 11, n. 4, p. 369-375, 2005.

CARVALHO, S.T.M. Propriedades Térmicas do Painel Aglomerado de Bagaço de Cana de Açúcar (Saccharum officinarum L.). Dissertação (Mestrado em Ciência e Tecnologia da Madeira). UFLN - Universidade Federal de Lavras. Lavras - MG. 66f. 2012.

FIORELLI, J.; LAHAR. F.A.R.; NASCIMENTO. M.F.; SAVASTANO JR, H.; ROSSIGNOLO. J.A. Painéis de partículas à base de bagaço de cana e resina de mamona - produção e propriedades. Act. Scien. Tec. v 33. p. 401-406, Maringá - PR. 2011.

IWAKIRI, S. Painéis de madeira reconstituída. FUPEF - Fundação de Pesquisas Florestais do Paraná. Curitiba, p.130-158. 2005.

MENDES, R.F.; GUIMARÃES JR, J.B.; SANTOS, R.C.; CÉSAR, A.A.S. Efeito da associação de bagaço de cana, do tipo e do teor de adesivo na produção de painéis aglomerados. Ciênc. Flor. Santa Maria. v. 22, n. 1, p. 161-170. 2012.

MOTA, S. Introdução à engenharia ambiental. Associação Brasileira de Engenharia Sanitária e Ambiental - ABES, Rio de Janeiro, 4ª edição, p. 343, 2006.

SILVA, O.G. Produção de etanol com a utilização do bagaço da cana de açúcar. Trabalho de Conclusão de Curso (Graduação) - Curso de Tecnologia em Biocombustíveis. Faculdade de Tecnologia de Araçatuba. Araçatuba. 41f . 2010. 
WEBER, C. Estudo sobre viabilidade de uso de resíduos de compensados, mdf e mdp para produção de painéis aglomerados. Dissertação (Mestrado em Ciências Florestais) - Universidade Federal do Paraná (UFPR). Curitiba - PR. 80f. 2011. 